\section{ХАРАКТЕРИСТИЧЕСКАЯ ЗАДАЧА ДЛЯ ОДНОГО ГИПЕРБОЛИЧЕСКОГО ДИФФЕРЕНЦИАЛЬНОГО УРАВНЕНИЯ ТРЕТЬЕГО ПОРЯДКА С НЕКРАТНЫМИ ХАРАКТЕРИСТИКАМИ}

\section{А. А. Андреев, Ю. О. Яковлева}

Самарский государственный технический университет E-mail: julia.yakovleva@mail.ru

В работе исследуется корректная, по Адамару, постановка характеристической задачи для одного гиперболического дифрференциального уравнения третьего порядка с некратными характеристиками.

Ключевые слова: гиперболическое дифрференциальное уравнение третьего порядка, некратные характеристики, характеристическая задача, корректность по Адамару.

The Characteristic Problem for one Hyperbolic Differentional Equation of the Third Order with Nonmultiple Characteristics

\section{A. A. Andreev, J. O. Yakovleva}

In the paper we consider the well-posed characteristics problem for the one hyperbolic differentional equation of the third order with the nonmultiple characteristics.

Key words: hyperbolic differentional equation of the third order, nonmultiple characteristics, characteristic problem, Hadamard's well-posedness.

\section{1. ПРЕДВАРИТЕЛЬНЫЕ СВЕДЕНИЯ}

Известно [1], что классическая задача Гурса для уравнения гиперболического типа второго порядка с двумя независимыми переменными с граничными условиями на двух характеристиках из различных семейств всегда является корректной по Адамару.

Исследованию начально-краевых задач для гиперболических уравнений и систем с двумя независимыми переменными порядка выше второго в случае кратных характеристик посвящены работы многих авторов. Например, в монографии А. В. Бицадзе [2] приведена характеристическая задача для систем второго порядка с кратными характеристиками. В статье С. С. Харибегашвили [3] рассмотрена характеристическая задача для вырождающихся гиперболических систем второго порядка. М. Х. Шхануковым [4] исследованы локальные и нелокальные краевые задачи для гиперболического уравнения третьего порядка. В статье А. П. Солдатова, М. Х. Шханукова [5] приведены краевые задачи с общим нелокальным условием для псевдопараболического уравнения высокого порядка. В [6] В. И. Жегаловым, Е. А. Уткиной также изучена характеристическая задача для одного псевдопараболического уравнения третьего порядка. О. М. Джохадзе [7] рассмотрена общая характеристическая задача типа Гурса для гиперболического уравнения третьего порядка с кратными характеристиками, а также сформулирована и исследована общая трехмерная характеристическая задача Гурса для линейных гиперболических уравнений третьего порядка с доминированными младшими членами [8]. О. С. Зикировым [9] исследована
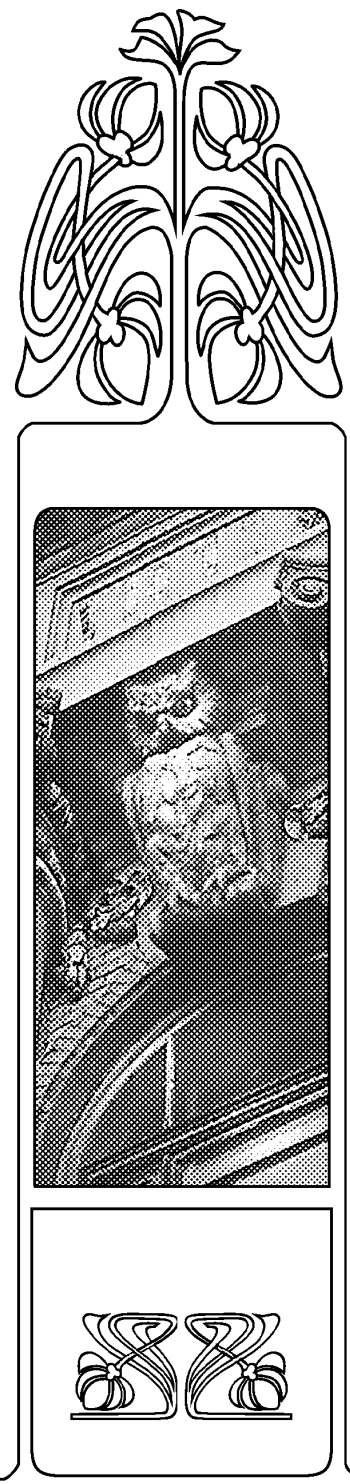

HАУЧНЫЙ
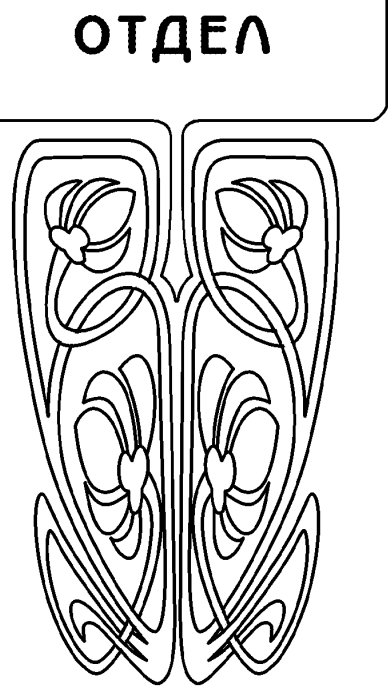
характеристическая задача Гурса для линейного гиперболического уравнения третьего порядка в прямоугольной области.

Характеристические задачи для систем и уравнений гиперболического типа в частных производных с некратными характеристиками изучены явно недостаточно. В монографии [2] приводятся примеры, показывающие, что для системы второго порядка с некратными характеристиками задача Гурса является некорректной по Адамару [10].

Целью нашей статьи является исследование корректности, по Адамару, характеристических задач для гиперболического уравнения от двух независимых переменных третьего порядка с некратными характеристиками.

В плоскости независимых переменных $x, y$ рассмотрим строго гиперболическое уравнение третьего порядка:

$$
u_{x x y}-u_{x y y}=0 .
$$

Лемма. Общее решение уравнения (1) из класса трижды непрерывно дифференцируемых функций $C^{3}(\mathbb{R})$ представляется в виде суммы

$$
u(x, y)=f\left(x-C_{1}\right)+g\left(y-C_{2}\right)+h\left(x+y-C_{3}\right)
$$

любых трех функций $f, g$ и $h$ из класса $C^{3}(\mathbb{R})$ от аргументов $x-C_{1}, y-C_{2}, x+y-C_{3}$ cоответственно, где $C_{1}, C_{2}, C_{3}-$ произвольные константы из $\mathbb{R}$.

Доказательство. Как известно, семейство линий $\varphi(x, y)=$ const является характеристиками уравнения (1), если функция $\varphi$ удовлетворяет дифференциальному уравнению:

$$
\frac{\partial^{3} \varphi}{\partial x^{2} \partial y}-\frac{\partial^{3} \varphi}{\partial x \partial y^{2}}=0
$$

Уравнение (3) равносильно дифференциальному уравнению:

$$
(d y)^{2} d x+d y(d x)^{2}=0 .
$$

Его решениями являются семейства линий, определяемые формулами

$$
x=C_{1}, \quad y=C_{2}, \quad x+y=C_{3} .
$$

Уравнение (1) допускает следующую факторизацию:

$$
\frac{\partial^{2}}{\partial x \partial y}(v(x, y))=0
$$

где $v(x, y)=u_{x}-u_{y}$.

Общее решение дифференциального уравнения первого порядка $u_{x}-u_{y}=F(x)+G(y)$ имеет вид

$$
u(x, y)=h(x+y)+\int_{0}^{x} F(t) d t+\int_{0}^{y} G(s) d s .
$$

Таким образом, получаем общее решение $u(x, y)$ в виде $(2)$. Лемма доказана.

Без ограничений общности можно считать, что общее решение уравнения (1) имеет вид

$$
u(x, y)=f(x)+g(y)+h(x+y) .
$$

Рассмотрим пример, иллюстрирующий некорректность классической постановки задачи Гурса на плоскости, независимых переменных $x, y$ для уравнений гиперболического типа третьего порядка.

Пример. Однородное уравнение (1), удовлетворяющее однородным условиям на характеристиках

$$
u(x, 0)=0, \quad x \in \mathbb{R}, \quad u(0, y)=0, \quad y \in \mathbb{R}, \quad u(x,-x)=0, \quad x \in \mathbb{R},
$$

имеет нетривиальное решение:

$$
u(x, y)=h(x+y)-h(x)-h(y), \quad h(0)=0,
$$

где $h(t) \in C^{3}(\mathbb{R})$ - любая нечетная функция.

Таким образом, нетривиальное решение (6) уравнения (1) удовлетворяет однородным граничным условиям (5) на трех характеристиках из различных семейств. В приведенной постановке характеристическая задача является некорректной по Адамару. 


\section{2. ХАРАКТЕРИСТИЧЕСКАЯ ЗАДАЧА НА ПЛОСКОСТИ}

Возникает вопрос: какая характеристическая задача будет являться корректной? Для уравнения (1) рассмотрим общую характеристическую задачу $G 1$.

Пусть $x \in I_{c}$, где $I_{c}$ имеет центральную симметрию, т. е. для любого $x \in I_{c} \quad 2 c-x \in I_{c}$, тогда для любой функции $f(x)$ справедливо

$$
f_{\mathcal{H}}^{c}=\frac{f(x)-f(2 c-x)}{2}, \quad f_{\mathcal{U}}^{c}=\frac{f(x)+f(2 c-x)}{2}, \quad f(x)=f_{\mathcal{H}}^{c}+f_{\mathcal{u}}^{c} .
$$

При $c=0$ будем обозначать $f_{\mathcal{H}}, f_{\mathcal{U}}$ соответственно.

Задача G1. Найти решение и $(x, y) \in C^{3}(\mathbb{R} \times \mathbb{R})$ уравнения (1), удовлетворяющее условиям

$$
u(x, 0)=\alpha(x), \quad x \in \mathbb{R}, \quad u(0, y)=\beta(y), \quad y \in \mathbb{R}, \quad u(x,-x)=\gamma(x), \quad x \in \mathbb{R},
$$

где $\alpha(x), \beta(y), \gamma(x) \in C^{3}(\mathbb{R})$.

Теорема 1. Если $\gamma_{H}=\alpha_{H}-\beta_{H}$, где $\alpha_{H}, \beta_{H}, \gamma_{H}-$ нечетнысе части функций $\alpha(x), \beta(x), \gamma(x)$ соответственно, то задача $G 1$ корректна по Адамару.

Определим функции $f, g$ и $h$ таким образом, чтобы удовлетворялись граничные условия (8), учитывая при этом условия согласования $f(0)+g(0)=\alpha(0)-h(0)$, получим:

$$
\begin{gathered}
f(x)=\alpha(x)-h(x)-g(0), \quad x \in \mathbb{R}, \\
g(y)=\beta(y)-h(y)-f(o), \quad y \in \mathbb{R}, \\
h(x)+h(-x)=\alpha(x)+\beta(-x)-\gamma(x)-\alpha(0)+2 h(0), \quad x \in \mathbb{R} .
\end{gathered}
$$

Тогда

$$
h(-x)+h(x)=\alpha(-x)+\beta(x)-\gamma(-x)-\alpha(0)+2 h(0), \quad x \in \mathbb{R} .
$$

Из (9) и (10) следует

$$
\begin{gathered}
\gamma_{\mathcal{H}}=\alpha_{\mathcal{H}}-\beta_{\mathcal{H}}, \\
h(x)=\frac{1}{2}\left[\alpha_{\mathcal{u}}(x)+\beta u(x)-\gamma u(x)-\alpha(0)+2 h(0)\right] .
\end{gathered}
$$

Подставляя (9), (11) и (12) в (4), получим:

$$
\begin{aligned}
& u(x, y)=\alpha(x)+\beta(y)-\frac{1}{2} \alpha(0)+\frac{1}{2}\left[\alpha_{\mathcal{u}}(x+y)-\alpha_{\mathcal{u}}(x)-\alpha_{\mathcal{u}}(y)\right]+ \\
& +\frac{1}{2}\left[\beta_{\mathcal{u}}(x+y)-\beta_{\mathcal{u}}(x)-\beta_{\mathcal{u}}(y)\right]-\frac{1}{2}\left[\gamma_{\mathcal{u}}(x+y)-\gamma_{\mathcal{u}}(x)-\gamma_{\mathcal{u}}(y)\right] .
\end{aligned}
$$

Формула (13) есть искомая функция, записанная в явном виде и являющаяся решением характеристической задачи $G 1$.

\section{3. ХАРАКТЕРИСТИЧЕСКАЯ ЗАДАЧА В ОБЛАСТИ, ОГРАНИЧЕННОЙ ХАРАКТЕРИСТИКАМИ}

Рассмотрим общую характеристическую задачу $G 2$ для уравнения (1) в области, ограниченной характеристиками.

Задача G2. Найти решение $и(x, y) \in C^{3}(\mathbb{R} \times \mathbb{R})$ уравнения (1), удовлетворяющее условиям

$$
u(x, 0)=\alpha(x), \quad 0 \leq x \leq 1, \quad u(0, y)=\beta(y), \quad 0 \leq y \leq 1, \quad u(x, 1-x)=\gamma(x), \quad 0 \leq x \leq 1,
$$

где $\alpha(x), \beta(y), \gamma(x) \in C^{3}(\mathbb{R})$.

Теорема 2. Если $\gamma_{H}^{\frac{1}{2}}=\alpha_{H}^{\frac{1}{2}}-\beta_{H}^{\frac{1}{2}}$, где $\alpha_{H}^{\frac{1}{2}}, \beta_{H}^{\frac{1}{2}}, \gamma_{H}^{\frac{1}{2}}-$ нечетные части функций $\alpha(x), \beta(x), \gamma(x)$, то задача $G 2$ корректна по Адамару.

Аналогично задаче $G 1$ получим:

$$
\begin{gathered}
u(x, y)=\alpha(x)+\beta(y)-\frac{1}{2} \alpha(0)+\frac{1}{2}\left[\alpha_{\mathcal{u}}^{\frac{1}{2}}(x+y)-\alpha_{\mathcal{u}}^{\frac{1}{2}}(x)-\alpha_{\mathcal{u}}^{\frac{1}{2}}(y)\right]+ \\
+\frac{1}{2}\left[\beta_{\mathcal{u}}^{\frac{1}{2}}(x+y)-\beta_{\mathfrak{u}}^{\frac{1}{2}}(x)-\beta_{\mathfrak{u}}^{\frac{1}{2}}(y)\right]-\frac{1}{2}\left[\gamma_{\underline{u}}^{\frac{1}{2}}(x+y)-\gamma_{\mathfrak{u}}^{\frac{1}{2}}(x)-\gamma_{\mathfrak{u}}^{\frac{1}{2}}(y)\right] .
\end{gathered}
$$


Формула (14) есть искомая функция, записанная в явном виде и являющаяся решением характеристической задачи $G 2$.

Нетрудно убедиться, что и в задаче $G 1$ и в задаче $G 2$ выбор характеристики, на которой задается видоизмененное условие, несущественен.

Отметим, что применение функциональных уравнений (7) с инволютивным сдвигом было предметом рассмотрения А. П. Хромова [11] и А. А. Андреева [12].

\section{Библиограсрический список}

1. Kyрант P. Уравнения с частными производными. M. : Мир, 1964. 831 с. [Courant R., Hilbert D. Methods of mathematical physics. Vol. II : Partial differential equations. New York; London : Interscience Publishers, 1962. 830 p.]

2. Бицадзе A. В. Некоторые классы уравнений в частных производных. М. : Наука, 1981. 448 с. [Bitsadze A. V. Some classes of partial differentional equations. Moscow : Nauka, 1981. 448 p.]

3. Харибегашвили C. С. О разрешимости одной характеристической задачи для вырождающихся гиперболических систем второго порядка // Дифференциальные уравнения. 1989. Т. 25, № 1. С. 154-162. [Kharibegashvili S. S. Solvability of a characteristic problem for second-order degenerate hyperbolic systems // Differ. Equ. 1989. Vol. 25, № 1. P. 123-131.]

4. Шхануков M. X. О некоторых краевых задачах для уравнения третьего порядка и экстремальных свойствах его решений //Дифференциальные уравнения. 1983. Т. 19, № 1. С. 145-152. [Soldatov A. P., Shkhanukov $M$. Kh. About some boundary value problems for third order equations // Differ. Equ. 1983. Vol. 19, № 1. P. 145-152.]

5. Солдатов А. П., Шхануков М. Х. Краевые задачи с общим нелокальным условием А. А. Самарского для псевдопараболических уравнений высокого порядка // Докл. АН СССР. 1987. Т. 297, № 3. С. 547552. [Soldatov A. P., Shkhanukov M. Kh. Boundary value problems with A. A. Samarski's general nonlocal condition for higher-order pseudoparabolic equations // Soviet Math. Dokl. 1988. Vol. 36, № 3. P. 507-511.]

6. Жегалов В. И., Уткина Е. А. Об одном псевдопараболическом уравнении третьего порядка // Изв. вузов. Математика. 1999. № 10 (449). С. 73-76. [Zhegalov V. I., Utkina E. A. Pseudoparabolic equation of the third order // Russian Math. (Izv. VUZ. Matematika). 1999. Vol. 43, № 10. Р. 70-73.]

7. Джохадзе О. М. Влияние младших членов на корректность постановки характеристических задач для гиперболических уравнений третьего порядка // Мат. заметки. 2003. Т. 74, № 4. С. 517-528. [Dzhokhadze O. M. Influence of lower terms on the well-posedness

of characteristics problems for third-order hyperbolic equations // Math. Notes. 2003. Vol. 74, № 4. P. 491501.]

8. Джохадзе О. М. О трехмерной обобщенной задаче Гурса для уравнения третьего порядка и связанные с ней общие двумерные интегральные уравнения вольтерры первого рода // Дифференциальные уравнения. 2006. T. 42, № 2. C. 385-394. [Dzhokhadze O. M. About the three-dimensional Goursat problem for third order differentional equations and related general-dimensional Volterra integral equations of the first kind // Differ. Equ. 2006. Vol. 42, № 2. Р. 385-394.]

9. Зикиров О. С. Локальные и нелокальные краевые задачи для гиперболических уравнений третьего порядка // Современная математика и ее приложения. 2011. Т. 68. С. 101-120. [Zikirov O. S. Local and nonlocal boundary-value problems for third-order hyperbolic equations // J. Math. Sci. Vol. 175, № 1. P. 104-123.]

10. Адамар Ж. Задача Коши для линейных уравнений с частными производными гиперболического типа. М. : Физматлит, 1994. 544 с. [Adamar J. Problem Cauchy for linear hyperbolic partial differential equations. Moscow : Phismathlit, 1994. 544 p.]

11. Хромов А. П. Смешанная задача для дифференциального уравнения с инволюцией и потенциалом специального вида // Изв. Сарат. ун-та. Нов. сер. Сер. Математика. Механика. Информатика. 2010. Т. 10, вып. 4. С. 17-22. [Khromov A. P. The mixed problem for the differential equation with involution and potential of the special kind // Izv. Saratov. Univer. New Series. Ser. Mathematics. Mechanics. Informatics. 2010. Vol. 10, iss. 4. P. 17-22.]

12. Андреев A. А. О корректности краевых задач для некоторых уравнений в частных производных с карлемановским сдвигом // Дифференциальные уравнения и их приложения : тр. 2-го Междунар. семинара. Самаpa : Изд-во Самар. ун-та, 1998. С. 5-18. [Andreev A. A. On the correctness of boundary value problems for some partial differential equations with a Carleman shift // Differential Equations and Their Applications : Proc. of the Second Intern. Seminar. Samara, 1998. P. 5-18.] 